\title{
Holmgren problem for Helmholtz equation with the three singular coefficients
}

\author{
Davlatjon R. Muydinjanov \\ Communicated by Ravshan Ashurov
}

\begin{abstract}
Fundamental solutions for a multidimensional Helmholtz equation with three singular coefficients have been constructed recently which are expressed in terms of the confluent hypergeometric function in four variables. In this paper, we study the Holmgren problem for a $3 \mathrm{D}$ elliptic equation with three singular coefficients. A unique solution of the problem is obtained in the explicit form.
\end{abstract}

Keywords. Holmgren problem, multidimensional elliptic equations with three singular coefficients, decomposition formulas, Lauricella hypergeometric function in three variables, confluent hypergeometric function in four variables.

2010 Mathematics Subject Classification. 35A08, 35J25,35J70,35J75.

\section{Introduction}

In the monograph of Gilbert [8], by applying a method of complex analysis, integral representation of solutions of the generalized bi-axially Helmholtz equation

$$
H_{\alpha \beta}^{\lambda}(u)=u_{x x}+u_{y y}+\frac{2 \alpha}{x} u_{x}+\frac{2 \beta}{y} u_{y}-\lambda^{2} u=0,0<2 \alpha, 2 \beta<1
$$

are constructed through analytic functions. The obtained formula contains rather bulky series and is inconvenient in applications. In article [2], the equation (1) was considered in two cases: when $\alpha=0, \beta>0$ and $\lambda=0, \beta>0$.

There are many works in which some problems for the modified equation (1) were studied [7, 15, 17, 20, 25]. In paper [9], for generalized bi-axially Helmholtz equation (1) four fundamental solutions in $R_{2}^{+}=\{(x, y): x>0, y>0\}$ were found and in works [21-23] the Neumann-Dirichlet type boundary value problems in the first quarter of the circle were solved.

Dirichlet and Dirichlet-Neumann (Holmgren) problems for elliptic equation with one singular coefficient in some part of ball were investigated by Agostinelli [1] and Olevskii [19]. Fundamental solutions for the following three (and more)- 
dimensional elliptic equations with two and three singular coefficients

$$
\begin{gathered}
u_{x x}+u_{y y}+u_{z z}+\frac{2 \alpha}{x} u_{x}+\frac{2 \beta}{y} u_{y}=0,0<2 \alpha, 2 \beta<1, \\
u_{x x}+u_{y y}+u_{z z}+\frac{2 \alpha}{x} u_{x}+\frac{2 \beta}{y} u_{y}+\frac{2 \gamma}{z} u_{z}=0,0<2 \alpha, 2 \beta, 2 \gamma<1
\end{gathered}
$$

and

$$
\begin{array}{r}
\sum_{i=1}^{p} u_{x_{i} x_{i}}+\frac{2 \alpha}{x_{1}} u_{x_{1}}+\frac{2 \beta}{x_{2}} u_{x_{2}}+\frac{2 \gamma}{x_{3}} u_{x_{3}}-\lambda^{2} u=0, \\
p \geq 3,0<2 \alpha, 2 \beta, 2 \gamma<1, \lambda \in R
\end{array}
$$

were constructed, respectively, in [14], [10] and [6], where $\alpha, \beta, \gamma$ and $\lambda$ are real numbers. For equations (2) and (3), the Dirichlet, Neumann and Holmgren problems were solved in some parts of the ball $[13,18,24]$.

In this paper, we study the Holmgren problem for the equation

$$
H_{\alpha \beta \gamma}^{\lambda}(u)=u_{x x}+u_{y y}+u_{z z}+\frac{2 \alpha}{x} u_{x}+\frac{2 \beta}{y} u_{y}+\frac{2 \gamma}{z} u_{z}-\lambda^{2} u=0,
$$

where $\alpha, \beta, \gamma$ and $\lambda$ are real numbers with $0<2 \alpha, 2 \beta, 2 \gamma<1$.

\section{Preliminaries}

Below, we give some formulas for Euler gamma-function, Gauss hypergeometric function, Lauricella hypergeometric function in three variables and a confluent hypergeometric function in four variables, which will be used in the next sections.

It is known that the Euler gamma-function $\Gamma(a)$ has the following properties [5, pp. 17-19, (2), (10), (15)]:

$$
\Gamma(a+m)=\Gamma(a)(a)_{m}, \quad \Gamma\left(a+\frac{1}{2}\right)=\frac{\sqrt{\pi} \Gamma(2 a)}{2^{2 a-1} \Gamma(a)}, \quad \Gamma\left(\frac{1}{2}\right)=\sqrt{\pi} .
$$

Here $(a)_{m}$ is a Pochhammer symbol, for which the equality

$$
(a)_{m+n}=(a)_{m}(a+m)_{n}
$$

is true $[5, \mathrm{p} .67,(5)]$.

A function

$$
F(a, b ; c ; x) \equiv F\left[\begin{array}{c}
a, b ; \\
c ;
\end{array}\right]:=\sum_{m=0}^{\infty} \frac{(a)_{m}(b)_{m}}{(c)_{m} m !} x^{m},|x|<1
$$


is known as the Gauss hypergeometric function and the equality

$$
\begin{array}{r}
F(a, b ; c ; 1)=\frac{\Gamma(c) \Gamma(c-a-b)}{\Gamma(c-a) \Gamma(c-b)}, \\
{[c \neq 0,-1,-2, \ldots ; \operatorname{Re}(c-a-b)>0]}
\end{array}
$$

holds [5, c.73, (14)]. Moreover, the following autotransformer formula [5, p.76, (22)]

$$
F(a, b ; c ; x)=(1-x)^{-b} F\left(c-a, b ; c ; \frac{x}{x-1}\right)
$$

is valid.

The confluent hypergeometric function in four variables has a form [6]

$$
\begin{gathered}
\mathrm{H}_{A}^{(3,1)}\left(a, b_{1}, b_{2}, b_{3} ; c_{1}, c_{2}, c_{3} ; x, y, z, t\right) \\
=\sum_{m, n, k, l=0}^{\infty} \frac{(a)_{m+n+k-l}\left(b_{1}\right)_{m}\left(b_{2}\right)_{n}\left(b_{3}\right)_{k}}{\left(c_{1}\right)_{m}\left(c_{2}\right)_{n}\left(c_{3}\right)_{k}} \frac{x^{m}}{m !} \frac{y^{n}}{n !} \frac{z^{k}}{k !} \frac{t^{l}}{l !},|x|+|y|+|z|<1 .
\end{gathered}
$$

The three-dimensional analogue of the function

$$
\mathrm{H}_{A}^{(3,1)}\left(a, b_{1}, b_{2}, b_{3} ; c_{1}, c_{2}, c_{3} ; x, y, z, t\right)
$$

was first introduced and investigated by Hasanov [9]:

$$
\begin{gathered}
A_{2}\left(a, b_{1}, b_{2} ; c_{1}, c_{2} ; x, y, t\right) \\
=\sum_{m, n, l=0}^{\infty} \frac{(a)_{m+n-l}\left(b_{1}\right)_{m}\left(b_{2}\right)_{n}}{\left(c_{1}\right)_{m}\left(c_{2}\right)_{n}} \frac{x^{m}}{m !} \frac{y^{n}}{n !} \frac{t^{l}}{l !},|x|+|y|<1 .
\end{gathered}
$$

For a given multiple hypergeometric function, it is useful to find a decomposition formula which would express the multivariable hypergeometric function in terms of products of several simpler hypergeometric functions involving fewer variables. Burchnall and Chaundy $[3,4]$ systematically presented a number of expansion and decomposition formulas for some double hypergeometric functions in series of simpler hypergeometric functions. For example, a Lauricella function in three variables is defined by [16]

$F_{A}^{(3)}\left(a, b_{1}, b_{2}, b_{3} ; c_{1}, c_{2}, c_{3} ; x, y, z\right)=\sum_{l, m, n=0}^{\infty} \frac{(a)_{l+m+n}\left(b_{1}\right)_{l}\left(b_{2}\right)_{m}\left(b_{1}\right)_{n}}{\left(c_{1}\right)_{l}\left(c_{2}\right)_{m}\left(c_{1}\right)_{n}} \frac{x^{l}}{l !} \frac{y^{m}}{m !} \frac{z^{n}}{n !}$

and the following decomposition formula holds true [11, 12]

$$
F_{A}^{(3)}\left(a ; b_{1}, b_{2}, b_{3} ; c_{1}, c_{2}, c_{3} ; x, y, z\right)
$$




$$
\begin{aligned}
=\sum_{l, m, n=0}^{\infty} & \frac{(a)_{l+m+n}\left(b_{1}\right)_{l+m}\left(b_{2}\right)_{l+n}\left(b_{3}\right)_{m+n}}{\left(c_{1}\right)_{l+m}\left(c_{2}\right)_{l+n}\left(c_{3}\right)_{m+n}} \frac{x^{l+m}}{n !} \frac{y^{l+n}}{m !} \frac{z^{m+n}}{l !} \\
& \times F\left(a+l+m, b_{1}+l+m ; c_{1}+l+m ; x\right) \\
& \times F\left(a+l+m+n, b_{2}+l+n ; c_{2}+l+n ; y\right) \\
\times & F\left(a+l+m+n, b_{3}+m+n ; c_{3}+m+n ; z\right) .
\end{aligned}
$$

Lemma 2.1. [6]. The following decomposition formula holds true

$$
\begin{gathered}
H_{A}^{(3,1)}\left(a ; b_{1}, b_{2}, b_{3} ; c_{1}, c_{2}, c_{3} ; x, y, z, t\right) \\
=\sum_{s=0}^{\infty} \sum_{q=0}^{s} \sum_{I(3, s)} A(s, q) C_{s}^{q} \frac{(-1)^{s+q}\left(b_{1}\right)_{i}\left(b_{2}\right)_{j}\left(b_{3}\right)_{k}}{(1-a)_{q}\left(c_{1}\right)_{i}\left(c_{2}\right)_{j}\left(c_{3}\right)_{k}} \frac{x^{i}}{i !} \frac{y^{j}}{j !} \frac{z^{k}}{k !} \frac{t^{q}}{q !} \\
\times F_{A}^{(3)}\left(a+s ; b_{1}+i, b_{2}+j, b_{3}+k ; c_{1}+i, c_{2}+j, c_{3}+k ; x, y, z\right) \\
\times_{0} F_{1}(1-a+q ;-t),
\end{gathered}
$$

where

$$
\begin{gathered}
A(s, q)=\left\{\begin{array}{c}
1, \text { if } s=0 \text { and } q=0, \\
q / s, \text { if } s \geq 1 \text { and } q \geq 0,
\end{array}\right. \\
I(3, s)=\{(i, j, k): i \geq 0, j \geq 0, k \geq 0, i+j+k=s\} .
\end{gathered}
$$

${ }_{0} F_{1}(a ; x)=\sum_{n=0}^{\infty} \frac{x^{n}}{(a)_{n} n !}$ is a generalized hypergeometric function [5, Chapter IV].

By virtue of the formulas (9) and (6), the expansion (10) yields

$$
\begin{gathered}
\mathrm{H}_{A}^{(3,1)}\left(a ; b_{1}, b_{2}, b_{3} ; c_{1}, c_{2}, c_{3} ; x, y, z, t\right)=(1-x)^{-b_{1}}(1-y)^{-b_{2}}(1-z)^{-b_{3}} \\
\times \sum_{l, m, n, s=0}^{\infty} \sum_{q=0}^{s} \sum_{I(3, s)} A(s, q) s ! C_{s}^{q} \\
\times \frac{(-1)^{s+q}(a)_{l+m+n+s}\left(b_{1}\right)_{i+l+m}\left(b_{2}\right)_{j+l+n}\left(b_{3}\right)_{k+m+n}}{(a)_{s}(1-a)_{q}\left(c_{1}\right)_{i+l+m}\left(c_{2}\right)_{j+l+n}\left(c_{3}\right)_{k+m+n} i ! j ! k ! n ! m ! l ! q !} t^{q} \\
\times\left(\frac{x}{1-x}\right)^{i+l+m} F\left(c_{1}-a-j-k, b_{1}+i+l+m ; c_{1}+i+l+m ; \frac{x}{x-1}\right) \\
\times\left(\frac{y}{1-y}\right)^{j+l+n} F\left(c_{2}-a-i-k-m, b_{2}+j+l+n ; c_{2}+j+l+n ; \frac{y}{y-1}\right) \\
\times\left(\frac{z}{1-z}\right)^{k+m+n} F\left(c_{3}-a-i-j-l, b_{3}+k+m+n ; c_{3}+k+m+n ; \frac{z}{z-1}\right) \\
\times{ }_{0} F_{1}(1-a+q ;-t) .
\end{gathered}
$$

Expansion (11) will be used for solving various boundary value problems for equation (4). 
In the present paper, $R_{3}^{3+}$ denotes $1 / 8$ part of the Euclidean space $R^{3}$ :

$$
R_{3}^{3+}:=\{(x, y, z): x>0, y>0, z>0,\} .
$$

All the fundamental solutions of equation (4) in the domain $R_{3}^{3+}$ were found in [6], and we will use one of these solutions in the study of the problem:

$$
q_{0}(x, y, z ; \xi, \eta, \zeta)=\gamma_{0} r^{-2 \alpha_{0}} \mathrm{H}_{\mathrm{A}}^{(3,1)}\left(\alpha_{0}, \alpha, \beta, \gamma ; 2 \alpha, 2 \beta, 2 \gamma ; \sigma\right),
$$

where

$$
\begin{gathered}
\sigma:=\left(\sigma_{1}, \sigma_{2}, \sigma_{3}, \sigma_{4}\right) \\
0<2 \alpha, 2 \beta, 2 \gamma<1, \quad \alpha_{0}=\frac{1}{2}+\alpha+\beta+\gamma \\
\gamma_{0}=2^{2 \alpha_{0}-3} \frac{\Gamma\left(\alpha_{0}\right)}{\pi^{3 / 2}} \frac{\Gamma(\alpha)}{\Gamma(2 \alpha)} \frac{\Gamma(\beta)}{\Gamma(2 \beta)} \frac{\Gamma(\gamma)}{\Gamma(2 \gamma)} \\
r^{2}=(x-\xi)^{2}+(y-\eta)^{2}+(z-\zeta)^{2}, \quad r_{1}^{2}=(x+\xi)^{2}+(y-\eta)^{2}+(z-\zeta)^{2} \\
r_{2}^{2}=(x-\xi)^{2}+(y+\eta)^{2}+(z-\zeta)^{2}, \quad r_{3}^{2}=(x-\xi)^{2}+(y-\eta)^{2}+(z+\zeta)^{2}
\end{gathered}
$$

and

$$
\sigma_{k}=1-\frac{r_{k}^{2}}{r^{2}}(k=1,2,3), \sigma_{4}=-\frac{\lambda^{2}}{4} r^{2}
$$

Here $\mathrm{H}_{\mathrm{A}}^{(3,1)}\left(\mathrm{a}, \mathrm{b}_{1}, \mathrm{~b}_{2}, \mathrm{~b}_{3} ; \mathrm{c}_{1}, \mathrm{c}_{2}, \mathrm{c}_{3} ; \mathrm{x}, \mathrm{y}, \mathrm{z}, \mathrm{t}\right)$ is a confluent hypergeometric function, defined in (7).

It is easy to verify that the fundamental solution $q_{0}(x, y, z ; \xi, \eta, \zeta)$ has the property

$$
\left.\left(x^{2 \alpha} \frac{\partial q_{0}}{\partial x}\right)\right|_{x=0}=0,\left.\left(y^{2 \alpha} \frac{\partial q_{0}}{\partial y}\right)\right|_{y=0}=0,\left.\quad\left(z^{2 \alpha} \frac{\partial q_{0}}{\partial z}\right)\right|_{z=0}=0
$$

\section{Formulation of the problem and uniqueness of the solution}

Let $\Omega \subset R_{3}^{+}$be a domain, bounded by planes

$$
\begin{aligned}
& S_{1}=\{(x, y, z): x=0,0<y<b, 0<z<c\}, \\
& S_{2}=\{(x, y, z): y=0,0<x<a, 0<z<c\}, \\
& S_{3}=\{(x, y, z): z=0,0<x<a, 0<y<b\}
\end{aligned}
$$

and by surface $S$ which intersects domains $S_{i}(i=\overline{1,3})$. Lines of intersections are designated as $\Gamma_{1}=S \cap S_{1}, \Gamma_{2}=S \cap S_{2}, \Gamma_{3}=S \cap S_{3}$ and $a, b, c=$ const. $>0$. 
Holmgren problem. Find a function $u(\mathrm{x}) \in C(\bar{\Omega}) \cap C^{2}(\Omega)$, satisfying equation (4) in $\Omega$ and conditions

$$
\begin{gathered}
\left.\left(x^{2 \alpha} \frac{\partial u}{\partial x}\right)\right|_{x=0}=\nu_{1}(y, z), \quad(y, z) \in S_{1}, \\
\left.\left(y^{2 \beta} \frac{\partial u}{\partial y}\right)\right|_{y=0}=\nu_{2}(x, z), \quad(x, z) \in S_{2}, \\
\left.\left(z^{2 \gamma} \frac{\partial u}{\partial z}\right)\right|_{z=0}=\nu_{3}(x, y), \quad(x, y) \in S_{3}, \\
\left.u\right|_{S}=\varphi(x, y, z), \quad(x, y, z) \in \bar{S},
\end{gathered}
$$

where $\nu_{k}$ and $\varphi$ are given functions, and, moreover, $\nu_{k}$ can reduce to an infinity of the order less than $1-2 \alpha-2 \beta-2 \gamma$ on the boundaries of $S_{k}$.

One can readily check that an equality

$$
\begin{gathered}
x^{2 \alpha} y^{2 \beta} z^{2 \gamma}\left[u H_{\alpha \beta \gamma}^{\lambda}(w)-w H_{\alpha \beta \gamma}^{\lambda}(u)\right]=\frac{\partial}{\partial x}\left[x^{2 \alpha} y^{2 \beta} z^{2 \gamma}\left(u w_{x}-w u_{x}\right)\right] \\
+\frac{\partial}{\partial y}\left[x^{2 \alpha} y^{2 \beta} z^{2 \gamma}\left(u w_{y}-w u_{y}\right)\right]+\frac{\partial}{\partial z}\left[x^{2 \alpha} y^{2 \beta} z^{2 \gamma}\left(u w_{z}-w u_{z}\right)\right]
\end{gathered}
$$

is true. Integrating both sides of above given equality on the domain $\Omega_{\varepsilon}$ and using the classical formula of Gauss-Ostrogradsky, we get

$$
\begin{aligned}
& \int_{\Omega_{\varepsilon}} x^{2 \alpha} y^{2 \beta} z^{2 \gamma}\left[u H_{\alpha \beta \gamma}^{\lambda}(w)-w H_{\alpha \beta \gamma}^{\lambda}(u)\right] d x d y d z \\
& \quad=\int_{\partial \Omega_{\varepsilon}} x^{2 \alpha} y^{2 \beta} z^{2 \gamma}\left(u w_{x}-w u_{x}\right) \cos (\mathbf{n}, x) d \vartheta \\
& \quad+\int_{\partial \Omega_{\varepsilon}} x^{2 \alpha} y^{2 \beta} z^{2 \gamma}\left(u w_{y}-w u_{y}\right) \cos (\mathbf{n}, y) d \vartheta \\
& \quad+\int_{\partial \Omega_{\varepsilon}} x^{2 \alpha} y^{2 \beta} z^{2 \gamma}\left(u w_{z}-w u_{z}\right) \cos (\mathbf{n}, z) d \vartheta
\end{aligned}
$$

Here $\Omega_{\varepsilon}$ be a sub-domain of $\Omega$ at a distance $\varepsilon>0$ from its boundary $\partial \Omega_{\varepsilon}=$ $S_{1} \cup S_{2} \cup S_{3} \cup S$ and

$$
\frac{\partial}{\partial \mathbf{n}}=\cos (\mathbf{n}, x) \cdot \frac{\partial}{\partial x}+\cos (\mathbf{n}, y) \cdot \frac{\partial}{\partial y}+\cos (\mathbf{n}, y) \cdot \frac{\partial}{\partial z},
$$

where $\mathbf{n}$ is outer normal to $\partial \Omega$. 
The following equality

$$
\begin{gathered}
\int_{\Omega_{\varepsilon}} x^{2 \alpha} y^{2 \beta} z^{2 \gamma} u H_{\alpha \beta \gamma}^{\lambda}(u) d x d y d z \\
+\int_{\Omega_{\varepsilon}} x^{2 \alpha} y^{2 \beta} z^{2 \gamma}\left[\left(\frac{\partial u}{\partial x}\right)^{2}+\left(\frac{\partial u}{\partial y}\right)^{2}+\left(\frac{\partial u}{\partial z}\right)^{2}+\lambda^{2} u^{2}\right] \\
=\int_{\Omega_{\varepsilon}}\left[\frac{\partial}{\partial x}\left(x^{2 \alpha} y^{2 \beta} z^{2 \gamma} u \frac{\partial u}{\partial x}\right)+\frac{\partial}{\partial y}\left(x^{2 \alpha} y^{2 \beta} z^{2 \gamma} u \frac{\partial u}{\partial y}\right)\right. \\
\left.+\frac{\partial}{\partial z}\left(x^{2 \alpha} y^{2 \beta} z^{2 \gamma} u \frac{\partial u}{\partial z}\right)\right] d x d y d z
\end{gathered}
$$

is valid. Applying the formula of Gauss-Ostrogradsky to this equality and letting $\varepsilon \rightarrow 0$, we get

$$
\begin{gathered}
\int_{\Omega_{\varepsilon}} x^{2 \alpha} y^{2 \beta} z^{2 \gamma}\left[\left(\frac{\partial u}{\partial x}\right)^{2}+\left(\frac{\partial u}{\partial y}\right)^{2}+\left(\frac{\partial u}{\partial z}\right)^{2}+\lambda^{2} u^{2}\right] d x d y d z \\
=-\int_{S_{1}} y^{2 \beta} z^{2 \gamma} \tau_{1} \nu_{1} d S_{1}-\int_{S_{2}} x^{2 \alpha} z^{2 \gamma} \tau_{2} \nu_{2} d S_{2} \\
-\int_{S_{3}} x^{2 \alpha} y^{2 \beta} \tau_{3} \nu_{3} d S_{3}-\int_{S} x^{2 \alpha} y^{2 \beta} z^{2 \gamma} \varphi \frac{\partial u}{\partial \mathbf{n}} d S
\end{gathered}
$$

where

$$
\tau_{1}:=\left.u(x, y, z)\right|_{x=0}, \tau_{2}:=\left.u(x, y, z)\right|_{y=0}, \tau_{3}:=\left.u(x, y, z)\right|_{z=0} .
$$

To prove the uniqueness of the solution, as usual, we suppose that the problem has two solutions $v$ and $w$. Denoting $u=v-w$, it satisfies homogeneous Holmgren problem $\left(\nu_{1}=0, \nu_{2}=0, \nu_{3}=0, \varphi=0\right)$. Further we have to prove that the homogeneous problem has only trivial solution. In this case, from (18) one can easily get

$$
\int_{\Omega_{\varepsilon}} x^{2 \alpha} y^{2 \beta} z^{2 \gamma}\left[\left(\frac{\partial u}{\partial x}\right)^{2}+\left(\frac{\partial u}{\partial y}\right)^{2}+\left(\frac{\partial u}{\partial z}\right)^{2}+\lambda^{2} u^{2}\right] d x d y d z=0 .
$$

Hence, it follows that $u_{x}=u_{y}=u_{z}=u=0$. We conclude that $u(x, y, z) \equiv 0$ in $\bar{\Omega}$. 


\section{Existence of the solution}

We prove the existence of the solution in a special case of the domain $\Omega$ in order to get the solution in an explicit form. With this aim, suppose that $a=b=c$ and $\mathrm{S}$ is a $1 / 8$ part of a sphere with radius $R=a$ and origin at the point $O(0,0,0)$. Let

$$
\Omega=\left\{(x, y, z): x^{2}+y^{2}+z^{2}<R^{2}, x>0, y>0, z>0\right\} .
$$

We find a solution of the considered problem using Green's function method [20]. Therefore, first we give the definition of Green's function for the formulated problem.

Definition 4.1. We call the function $G(x, y, z ; \xi, \eta, \zeta)$ as Green's function of the Holmgren problem, if it satisfies the following conditions:

- this function is a regular solution of equation (4) in the domain $\Omega$, expect at the point $(\xi, \eta, \zeta)$, which is any fixed point of $\Omega$,

- it satisfies boundary conditions

$$
\begin{gathered}
\left.\left(x^{2 \alpha} \frac{\partial G(x, y, z ; \xi, \eta, \zeta)}{\partial x}\right)\right|_{x=0}=0,\left.\left(y^{2 \beta} \frac{\partial G(x, y, z ; \xi, \eta, \zeta)}{\partial y}\right)\right|_{y=0}=0, \\
\left.\left(z^{2 \gamma} \frac{\partial G(x, y, z ; \xi, \eta, \zeta)}{\partial z}\right)\right|_{z=0}=0,\left.\quad G(x, y, z ; \xi, \eta, \zeta)\right|_{S}=0,
\end{gathered}
$$

- it can be represented as

$$
G(x, y, z ; \xi, \eta, \zeta)=q_{0}(x, y, z ; \xi, \eta, \zeta)+q_{0}^{*}(x, y, z ; \xi, \eta, \zeta),
$$

where $q_{0}(x, y, z ; \xi, \eta, \zeta)$ is the fundamental solution found earlier (see formula (12)) and the function

$$
q_{0}^{*}(x, y, z ; \xi, \eta, \zeta)=-\left(\frac{a}{R_{0}}\right)^{2 \alpha_{0}} q_{0}(x, y, z ; \bar{\xi}, \bar{\eta}, \bar{\zeta})
$$

is a regular solution of equation (4) in the domain $\Omega$. Here,

$$
\bar{\xi}=\frac{a^{2}}{R_{0}^{2}} \xi, \quad \bar{\eta}=\frac{a^{2}}{R_{0}^{2}} \eta, \quad \bar{\zeta}=\frac{a^{2}}{R_{0}^{2}} \zeta, \quad R_{0}^{2}=\xi^{2}+\eta^{2}+\zeta^{2} .
$$

Excise a small ball with its center at $(\xi, \eta, \zeta)$ and with radius $\rho>0$ from the domain $\Omega$. Designate the sphere of the excised ball as $C_{\rho}$ and by $\Omega_{\rho}$ denote the 
remaining part of $\Omega$.

Applying formula (18), we obtain

$$
\begin{gathered}
\int_{C_{\rho}} x^{2 \alpha} y^{2 \beta} z^{2 \gamma}\left[u \frac{\partial G}{\partial \mathbf{n}}-G \frac{\partial u}{\partial \mathbf{n}}\right] d C_{\rho} \\
=-\int_{S_{1}} y^{2 \beta} z^{2 \gamma} G(0, y, z ; \xi, \eta, \zeta) \nu_{1} d S_{1}-\int_{S_{2}} x^{2 \alpha} z^{2 \gamma} G(x, 0, z ; \xi, \eta, \zeta) \nu_{2} d S_{2} \\
-\int_{S_{3}} x^{2 \alpha} y^{2 \beta} G(x, y, 0 ; \xi, \eta, \zeta) \nu_{3} d S_{3}-\int_{S} x^{2 \alpha} y^{2 \beta} z^{2 \gamma} \varphi \frac{\partial u}{\partial \mathbf{n}} d S .
\end{gathered}
$$

First, we consider the integral

$$
\int_{C_{\rho}} x^{2 \alpha} y^{2 \beta} z^{2 \gamma} u \frac{\partial G}{\partial \mathbf{n}} d C_{\rho} .
$$

Taking (19) into account we rewrite it as follows

$$
\begin{gathered}
\int_{C_{\rho}} x^{2 \alpha} y^{2 \beta} z^{2 \gamma} u \frac{\partial G}{\partial \mathbf{n}} d C_{\rho} \equiv \int_{C_{\rho}} x^{2 \alpha} y^{2 \beta} z^{2 \gamma} u \frac{\partial q_{0}(x, y, z ; \xi, \eta, \zeta)}{\partial \mathbf{n}} d C_{\rho} \\
+\int_{C_{\rho}} x^{2 \alpha} y^{2 \beta} z^{2 \gamma} u \frac{\partial q_{0}^{*}(\mathrm{x}, \mathrm{y}, \mathrm{z} ; \xi, \eta, \zeta)}{\partial \mathbf{n}} d C_{\rho} \equiv I_{1}+I_{2} .
\end{gathered}
$$

Using the differentiation formula, we get

$$
\begin{aligned}
& \frac{\partial^{i+j+k+l}}{\partial \sigma_{1}^{i} \partial \sigma_{2}^{j} \partial \sigma_{3}^{k} \partial \sigma_{4}^{l}} \mathrm{H}_{A}^{(3,1)}\left(a, b_{1}, b_{2}, b_{3} ; c_{1}, c_{2}, c_{3} ; \sigma\right)=\frac{(a)_{i+j+k-l}\left(b_{1}\right)_{i}\left(b_{2}\right)_{j}\left(b_{3}\right)_{k}}{\left(c_{1}\right)_{i}\left(c_{2}\right)_{j}\left(c_{3}\right)_{k}} \\
& \times \mathrm{H}_{A}^{(3,1)}\left(a+i+j+k-l, b_{1}+i, b_{2}+j, b_{3}+k ; ; c_{1}+i, c_{2}+j, c_{3}+k ; \sigma\right)
\end{aligned}
$$

and the following an adjacent relation

$$
\begin{aligned}
& \frac{a b_{1}}{c_{1}} \sigma_{1} \mathrm{H}_{A}^{(3,1)}\left(a+1, b_{1}+1, b_{2}, b_{3} ; c_{1}+1, c_{2}, c_{3} ; \sigma\right) \\
& +\frac{a b_{2}}{c_{2}} \sigma_{2} \mathrm{H}_{A}^{(3,1)}\left(a+1, b_{1}, b_{2}+1, b_{3} ; c_{1}, c_{2}+1, c_{3} ; \sigma\right) \\
& +\frac{a b_{3}}{c_{3}} \sigma_{3} \mathrm{H}_{A}^{(3,1)}\left(a+1, b_{1}, b_{2}, b_{3}+1 ; c_{1}, c_{2}, c_{3}+1 ; \sigma\right) \\
& -\frac{1}{a-1} \sigma_{4} \mathrm{H}_{A}^{(3,1)}\left(a-1, b_{1}, b_{2}, b_{3} ; c_{1}, c_{2}, c_{3} ; \sigma\right) \\
& =a \mathrm{H}_{A}^{(3,1)}\left(a+1, b_{1}, b_{2}, b_{3} ; c_{1}, c_{2}, c_{3} ; \sigma\right)-a \mathrm{H}_{A}^{(3,1)}\left(a, b_{1}, b_{2}, b_{3} ; c_{1}, c_{2}, c_{3} ; \sigma\right) .
\end{aligned}
$$


We define

$$
\frac{\partial q_{0}}{\partial \mathbf{n}}=\frac{\partial q_{0}}{\partial x} \cdot \cos (\mathbf{n}, x)+\frac{\partial q_{0}}{\partial y} \cdot \cos (\mathbf{n}, y)+\frac{\partial q_{0}}{\partial z} \cdot \cos (\mathbf{n}, z) .
$$

Indeed, substituting following three formulas of differentiation

$$
\begin{aligned}
& \frac{\partial q_{0}(x, y, z ; \xi, \eta, \zeta)}{\partial x} \\
& =-2 \alpha_{0} \gamma_{0} \xi r^{-2 \alpha_{0}-2} \mathrm{H}_{A}^{(3,1)}\left(\alpha_{0}+1,1+\alpha, \beta, \gamma ; 1+2 \alpha, 2 \beta, 2 \gamma ; \sigma\right) \\
& -2 \alpha_{0} \gamma_{0}(x-\xi) r^{-2 \alpha_{0}-2} \mathrm{H}_{A}^{(3,1)}\left(\alpha_{0}+1, \alpha, \beta, \gamma ; 2 \alpha, 2 \beta, 2 \gamma ; \sigma\right), \\
& \frac{\partial q_{0}(x, y, z ; \xi, \eta, \zeta)}{\partial y} \\
& =-2 \alpha_{0} \gamma_{0} \eta r^{-2 \alpha_{0}-2} \mathrm{H}_{A}^{(3,1)}\left(\alpha_{0}+1, \alpha, 1+\beta, \gamma ; 2 \alpha, 1+2 \beta, 2 \gamma ; \sigma\right) \\
& -2 \alpha_{0} \gamma_{0}(y-\eta) r^{-2 \alpha_{0}-2} \mathrm{H}_{A}^{(3,1)}\left(\alpha_{0}+1, \alpha, \beta, \gamma ; 2 \alpha, 2 \beta, 2 \gamma ; \sigma\right), \\
& \frac{\partial q_{0}(x, y, z ; \xi, \eta, \zeta)}{\partial z} \\
& =-2 \alpha_{0} \gamma_{0} \zeta r^{-2 \alpha_{0}-2} \mathrm{H}_{A}^{(3,1)}\left(\alpha_{0}+1, \alpha, \beta, 1+\gamma ; 2 \alpha, 2 \beta, 1+2 \gamma ; \sigma\right) \\
& -2 \alpha_{0} \gamma_{0}(z-\zeta) r^{-2 \alpha_{0}-2} \mathrm{H}_{A}^{(3,1)}\left(\alpha_{0}+1, \alpha, \beta, \gamma ; 2 \alpha, 2 \beta, 2 \gamma ; \sigma\right) \\
& , w g e t
\end{aligned}
$$

in (21), we get

$$
\begin{aligned}
& \frac{\partial q_{0}}{\partial \mathbf{n}}=-\alpha_{0} \gamma_{0} r^{-2 \alpha_{0}} \mathbf{H}_{A}^{(3,1)}\left(\alpha_{0}+1, \alpha, \beta, \gamma ; 2 \alpha, 2 \beta, 2 \gamma ; \sigma\right) \frac{\partial}{\partial \mathbf{n}}\left[\ln r^{2}\right] \\
& +\Phi(x, y, z ; \xi, \eta, \zeta),
\end{aligned}
$$

where

$$
\begin{aligned}
& \Phi(x, y, z ; \xi, \eta, \zeta) \\
& =-2 \alpha_{0} \gamma_{0} \xi r^{-2 \alpha_{0}-2} \mathrm{H}_{A}^{(3,1)}\left(\alpha_{0}+1,1+\alpha, \beta, \gamma ; 1+2 \alpha, 2 \beta, 2 \gamma ; \sigma\right) \cos (\mathbf{n} ; x) \\
& -2 \alpha_{0} \gamma_{0} \eta r^{-2 \alpha_{0}-2} \mathrm{H}_{A}^{(3,1)}\left(\alpha_{0}+1, \alpha, 1+\beta, \gamma ; 2 \alpha, 1+2 \beta, 2 \gamma ; \sigma\right) \cos (\mathbf{n} ; y) \\
& -2 \alpha_{0} \gamma_{0} \zeta r^{-2 \alpha_{0}-2} \mathrm{H}_{A}^{(3,1)}\left(\alpha_{0}+1, \alpha, \beta, 1+\gamma ; 2 \alpha, 2 \beta, 1+2 \gamma ; \sigma\right) \cos (\mathbf{n} ; z) .
\end{aligned}
$$

We separate the left part of identity (20) on three integrals

$$
\int_{C_{\rho}} x^{2 \alpha} y^{2 \beta} z^{2 \gamma} u \frac{\partial G}{\partial \mathbf{n}} d C_{\rho}=J_{1}+J_{2}+J_{3},
$$

where

$$
\begin{aligned}
J_{1}= & -\alpha_{0} \gamma_{0} \int_{C_{\rho}} x^{2 \alpha} y^{2 \beta} z^{2 \gamma} u r^{-2 \alpha_{0}} \\
& \times \mathrm{H}_{A}^{(3,1)}\left(\alpha_{0}+1, \alpha, \beta, \gamma ; 2 \alpha, 2 \beta, 2 \gamma ; \sigma\right) \frac{\partial}{\partial \mathbf{n}}\left[\ln r^{2}\right] d C_{\rho},
\end{aligned}
$$




$$
\begin{gathered}
J_{2}=\int_{C_{\rho}} x^{2 \alpha} y^{2 \beta} z^{2 \gamma} u \Phi(x, y, z ; \xi, \eta, \zeta) d C_{\rho}, \\
J_{3}=\int_{C_{\rho}} x^{2 \alpha} y^{2 \beta} z^{2 \gamma} u \frac{\partial q_{1}^{*}}{\partial \mathbf{n}} d C_{\rho} .
\end{gathered}
$$

We use the following spherical system of coordinates [20]:

$$
\begin{gathered}
x=\xi+\rho \cos \varphi, y=\eta+\rho \sin \varphi \cos \psi, z=\zeta+\rho \sin \varphi \sin \psi, \\
{[\rho \geq 0,0 \leq \varphi \leq \pi, 0 \leq \psi \leq 2 \pi] .}
\end{gathered}
$$

Then, we have

$$
\begin{gathered}
J_{1}=2 \alpha_{0} \gamma_{0} \rho^{-2 \alpha_{0}+1} \int_{0}^{2 \pi} d \psi \int_{0}^{\pi}(\xi+\rho \cos \varphi)^{2 \alpha} \\
\times(\eta+\rho \sin \varphi \cos \psi)^{2 \beta}(\zeta+\rho \sin \varphi \sin \psi)^{2 \gamma} \\
\times u(\xi+\rho \cos \varphi, \eta+\rho \sin \varphi \cos \psi, \zeta+\rho \sin \varphi \sin \psi) \\
\times \mathrm{H}_{\mathrm{A}}^{(3,1)}\left(\alpha_{0}+1, \alpha, \beta, \gamma ; 2 \alpha, 2 \beta, 2 \gamma ; \sigma_{1 \rho}, \sigma_{2 \rho}, \sigma_{3 \rho}, \sigma_{4 \rho}\right) \sin \varphi \mathrm{d} \varphi,
\end{gathered}
$$

where

$$
\begin{gathered}
\sigma_{1 \rho}=-\frac{4(\xi+\rho \cos \varphi) \xi}{\rho^{2}}, \sigma_{2 \rho}=-\frac{4(\eta+\rho \sin \varphi \cos \psi) \eta}{\rho^{2}} \\
\sigma_{3 \rho}=-\frac{4(\zeta+\rho \sin \varphi \sin \psi)}{\rho^{2}}, \sigma_{4 \rho}=-\frac{\lambda^{2}}{4} \rho^{2} .
\end{gathered}
$$

First we evaluate $\mathrm{H}_{\mathrm{A}}^{(3,1)}$. Using the decomposition formula (11), we find

$$
\begin{aligned}
\mathrm{H}_{\mathrm{A}}^{(3,1)}\left(\alpha_{0}\right. & \left.+1, \alpha, \beta, \gamma ; 2 \alpha, 2 \beta, 2 \gamma ; \sigma_{1 \rho}, \sigma_{2 \rho}, \sigma_{3 \rho}, \sigma_{4 \rho}\right) \\
& =\rho^{2 \alpha+2 \beta+2 \gamma} r_{1 \rho}^{-2 \alpha} r_{2 \rho}^{-2 \beta} r_{3 \rho}^{-2 \gamma} \cdot \aleph
\end{aligned}
$$

where

$$
\begin{gathered}
r_{1 \rho}^{2}=(2 \xi+\rho \cos \varphi)^{2}+\rho^{2} \sin ^{2} \varphi, \\
r_{2 \rho}^{2}=\rho^{2} \cos ^{2} \varphi+(2 \eta+\rho \sin \varphi \cos \psi)^{2}+\rho^{2} \sin ^{2} \varphi \sin ^{2} \psi, \\
r_{3 \rho}^{2}=\rho^{2} \cos ^{2} \varphi+\rho^{2} \sin ^{2} \varphi \cos ^{2} \psi+(2 \zeta+\rho \sin \varphi \cos \psi)^{2}, \\
\aleph=\sum_{l, m, n, s=0}^{\infty} \sum_{q=0}^{s} \sum_{I(3 . s)} A(s, q) s ! C_{s}^{q}
\end{gathered}
$$




$$
\begin{aligned}
& \times \frac{(-1)^{s+q}\left(1+\alpha_{0}\right)_{l+m+n+s}(\alpha)_{i+l+m}(\beta)_{j+l+n}(\gamma)_{k+m+n}}{\left(1+\alpha_{0}\right)_{s}\left(-\alpha_{0}\right)_{q}(2 \alpha)_{i+l+m}(2 \beta)_{j+l+m}(2 \gamma)_{k+m+n} k ! n ! m ! q !} \\
& \times\left(\frac{\rho^{2}}{r_{1 \rho}^{2}}-1\right)^{i+l+m} F\left[\begin{array}{cc}
2 \alpha-\alpha_{0}-1-j-k, \alpha+i+l+m ; & 1-\frac{\rho^{2}}{r_{1 \rho}^{2}}
\end{array}\right] \\
& \times\left(\frac{\rho^{2}}{r_{2 \rho}^{2}}-1\right)^{j+l+n} F\left[\begin{array}{cc}
2 \beta-\alpha_{0}-1-i-k-m, \beta+j+l+n ; & 1-\frac{\rho^{2}}{r_{2 \rho}^{2}}
\end{array}\right] \\
& \times\left(\frac{\rho^{2}}{r_{3 \rho}^{2}}-1\right)^{k+m+n} F\left[\begin{array}{c}
2 \gamma-\alpha_{0}-1-i-j-l, \gamma+k+m+n ; \\
2 \gamma+k+m+n ;
\end{array} \quad \frac{\rho^{2}}{r_{3 \rho}^{2}}\right] \\
& \times{ }_{0} F_{1}\left(-\alpha_{0}+q ;-\frac{\lambda^{2}}{4} \rho\right) .
\end{aligned}
$$

It is easy to see that when $\rho \rightarrow 0$, the function $\aleph$ becomes an expression that does not depend on $x, y, z$ and $\xi, \eta, \zeta$ :

$$
\begin{aligned}
& \lim _{\rho \rightarrow 0} \aleph=\sum_{l, m, n=0}^{\infty} \frac{\left(\alpha_{0}+1\right)_{l+m+n}(\alpha)_{l+m}(\beta)_{l+n}(\gamma)_{m+n}}{(2 \alpha)_{l+m}(2 \beta)_{l+n}(2 \gamma)_{m+n} m ! n ! l !} \\
& \quad \times F\left(2 \alpha-\alpha_{0}-1, \alpha+l+m ; 2 \alpha+l+m ; 1\right) \\
& \quad \times F\left(2 \beta-\alpha_{0}-1-m, \beta+l+n ; 2 \beta+l+n ; 1\right) \\
& \quad \times F\left(2 \gamma-\alpha_{0}-1-l, \gamma+m+n ; 2 \gamma+m+n ; 1\right) .
\end{aligned}
$$

Now, applying summation formula (5) to each hypergeometric function $F(a, b, c ; 1)$ in the last sum, we get

$$
\lim _{\rho \rightarrow 0} \aleph=\Gamma\left(\frac{3}{2}\right) \frac{\Gamma(2 \alpha) \Gamma(2 \beta) \Gamma(2 \gamma)}{\Gamma\left(\alpha_{0}+1\right) \Gamma(\alpha) \Gamma(\beta) \Gamma(\gamma)} .
$$

If we choose $\gamma_{0}$ as (13), we then have

$$
\lim _{\rho \rightarrow 0} J_{1}=u(\xi, \eta, \zeta)
$$

By similar evaluations, one can get that

$$
\lim _{\rho \rightarrow 0} J_{2}=\lim _{\rho \rightarrow 0} J_{3}=0 .
$$


If we consider the integral

$$
\int_{C_{\rho}} x^{2 \alpha} y^{2 \beta} z^{2 \gamma} \cdot G(x, \xi) \frac{\partial u}{\partial n} d C_{\rho},
$$

and use the algorithm given above for evaluations, we can prove that

$$
\lim _{\rho \rightarrow 0} \int_{C_{\rho}} x^{2 \alpha} y^{2 \beta} z^{2 \gamma} \cdot G(x, \xi) \frac{\partial u}{\partial n} d C_{\rho}=0 .
$$

From (20), we can write the solution of the Holmgren problem as follows:

$$
\begin{gathered}
u(\xi, \eta, \zeta)=-\int_{S_{1}} y^{2 \beta} z^{2 \gamma} G_{1}^{*}(y, z ; \xi, \eta, \zeta) \nu_{1}(y, z) d S_{1} \\
-\int_{S_{2}} x^{2 \alpha} z^{2 \gamma} G_{2}^{*}(x, z ; \xi, \eta, \zeta) \nu_{2}(x, z) d S_{2}-\int_{S_{3}} x^{2 \alpha} y^{2 \beta} G_{2}^{*}(x, y ; \xi, \eta, \zeta) \nu_{3}(x, y) d S_{3} \\
-\int_{S} x^{2 \alpha} y^{2 \beta} z^{2 \gamma} \frac{\partial G(x, y, z ; \xi, \eta, \zeta)}{\partial n} \varphi(x, y, z) d S .
\end{gathered}
$$

The particular values of Green's function are given by

$$
\begin{gathered}
G_{1}^{*}(y, z ; \xi, \eta, \zeta)=2 \alpha_{0} \gamma_{1}\left\{\frac{A_{2}\left(\alpha_{0}, \beta, \gamma, 2 \beta, 2 \gamma ; \eta_{01}^{(x)}, \zeta_{01}^{(x)}, \theta_{01}^{(x)}\right)}{\left[\xi^{2}+(\eta-y)^{2}+(\zeta-z)^{2}\right]^{\alpha_{0}}}\right. \\
\left.-\frac{A_{2}\left(\alpha_{0}, \beta, \gamma, 2 \beta, 2 \gamma ; \eta_{02}^{(x)}, \zeta_{02}^{(x)}, \theta_{02}^{(x)}\right)}{\left[\left(a-\frac{y \eta}{a}\right)^{2}+\left(a-\frac{z \zeta}{a}\right)^{2}+\frac{\xi^{2}+\zeta^{2}}{a^{2}} y^{2}+\frac{\xi^{2}+\eta^{2}}{a^{2}} z^{2}-a^{2}\right]^{\alpha_{0}}}\right\}, \\
G_{2}^{*}(x, z ; \xi, \eta, \zeta)=2 \alpha_{0} \gamma_{1}\left\{\frac{A_{2}\left(\alpha_{0}, \alpha, \gamma, 2 \alpha, 2 \gamma ; \xi_{01}^{(y)}, \zeta_{01}^{(y)}, \theta_{01}^{(y)}\right.}{\left[(\xi-x)^{2}+\eta^{2}+(\zeta-z)^{2}\right]^{\alpha_{0}}}\right. \\
\left.-\frac{\left[\left(\alpha_{0}, \alpha, \gamma, 2 \alpha, 2 \gamma ; \xi_{02}^{(y)}, \zeta_{02}^{(y)}, \theta_{02}^{(y)}\right)\right.}{\left[\left(a-\frac{x \xi}{a}\right)^{2}+\left(a-\frac{z \zeta}{a}\right)^{2}+\frac{\eta^{2}+\zeta^{2}}{a^{2}} x^{2}+\frac{\xi^{2}+\eta^{2}}{a^{2}} z^{2}-a^{2}\right]^{\alpha_{0}}}\right\},
\end{gathered}
$$




$$
\begin{gathered}
G_{3}^{*}(x, y ; \xi, \eta, \zeta)=2 \alpha_{0} \gamma_{1}\left\{\frac{A_{2}\left(\alpha_{0}, \alpha, \beta, 2 \alpha, 2 \beta ; \xi_{01}^{(z)}, \eta_{01}^{(z)}, \theta_{01}^{(z)}\right)}{\left[(\xi-x)^{2}+(\eta-y)^{2}+\zeta^{2}\right]^{\alpha_{0}}}\right. \\
\left.-\frac{A_{2}\left(\alpha_{0}, \alpha, \beta, 2 \alpha, 2 \beta ; \xi_{02}^{(z)}, \eta_{02}^{(z)}, \theta_{02}^{(z)}\right)}{\left[\left(a-\frac{x \xi}{a}\right)^{2}+\left(a-\frac{y \eta}{a}\right)^{2}+\frac{\eta^{2}+\zeta^{2}}{a^{2}} x^{2}+\frac{\xi^{2}+\zeta^{2}}{a^{2}} y^{2}-a^{2}\right]^{\alpha_{0}}}\right\},
\end{gathered}
$$

where

$$
\begin{gathered}
\xi_{0 i}^{(y)}=\left.\xi_{i}\right|_{y=0}, \xi_{0 i}^{(z)}=\left.\xi_{i}\right|_{z=0}, \eta_{0 i}^{(x)}=\left.\eta_{i}\right|_{x=0}, \eta_{0 i}^{(z)}=\left.\eta_{i}\right|_{z=0}, \\
\zeta_{0 i}^{(x)}=\left.\zeta_{i}\right|_{x=0}, \zeta_{0 i}^{(y)}=\left.\zeta_{i}\right|_{y=0}, \xi_{i}=\mu_{i} x \xi, \eta_{i}=\mu_{i} y \eta, \zeta_{i}=\mu_{i} z \zeta, \\
\theta_{0 i}^{(x)}=\left.\theta_{i}\right|_{x=0}, \theta_{0 i}^{(y)}=\left.\theta_{i}\right|_{y=0}, \theta_{0 i}^{(z)}=\left.\theta_{i}\right|_{z=0}, i=1,2 \\
\mu_{1}=-\frac{4}{r^{2}}, \mu_{2}=-\frac{a^{2}}{R_{0}^{2}} \frac{4}{R^{2}}, \theta_{1}=-\frac{\lambda^{2}}{4} r^{2}, \theta_{2}=-\frac{\lambda^{2}}{4} \frac{a^{2}}{R_{0}^{2}} R^{2}, \\
r^{2}=(\xi-x)^{2}+(\eta-y)^{2}+(\zeta-z)^{2}, \\
R^{2}=\left(a-\frac{x \xi}{a}\right)^{2}+\left(a-\frac{y \eta}{a}\right)^{2}+\left(a-\frac{z \zeta}{a}\right)^{2} \\
+\frac{\eta^{2}+\zeta^{2}}{a^{2}} x^{2}+\frac{\xi^{2}+\zeta^{2}}{a^{2}} y^{2}+\frac{\xi^{2}+\eta^{2}}{a^{2}} z^{2}-2 a^{2}
\end{gathered}
$$

Here, $A_{2}\left(a, b_{1}, b_{2} ; c_{1}, c_{2} ; x, y, t\right)$ is confluent hypergeometric function, defined in (8).

Thus, the following theorem is proved.

Theorem 4.2. The solution of the Holmgren problem with conditions (14)-(17) exists and is defined by formula (22). 


\section{Bibliography}

[1] C. Agostinelli, Integrazione dell'equazione differenziale $\frac{\partial u}{\partial x^{2}}+\frac{\partial u}{\partial y^{2}}+\frac{\partial u}{\partial z^{2}}+\frac{1}{x} u_{x}=f$ e problema analogo a quello di Dirichlet per un campo emisferico, Atti Accad. Naz. Lincei, Rend., VI. Ser. 26 (1937), 138-143 (in Italian).

[2] A. Altin, Solutions of type $r^{m}$ for a class of singular equations, Internat. J. Math. Math. Sci. 5(3) (1982), 613-619.

[3] J. L. Burchnall and T. W. Chaundy, Expansions of Appell's double hypergeometric functions, Quart. J. Math. Oxford 11 (1940), 249-270.

[4] J. L. Burchnall and T. W. Chaundy, Expansions of Appell's double hypergeometric functions II, Quart. J. Math. Oxford 12 (1941), 112-128.

[5] A. Erdelyi, W. Magnus, F. Oberhettinger and F. G. Tricomi, Higher Transcendental Functions, Vol.I, McGraw-Hill, New York, Toronto and London, 1953.

[6] T. G. Ergashev, On fundamental solutions for multidimensional Helmholtz equation with three singular coefficients, Computers and Mathematics with Applications 77 (2019), 69-76.

[7] A. J. Fryant, Growth and complete sequences of generalized bi-axially symmetric potentials, J. Differ. Equations 31(2) (1979), 155-164.

[8] R. Gilbert, Function Theoretic Methods in Partial Differential Equations, Academic Press, New York, London, 1969.

[9] A. Hasanov, Fundamental solutions of generalized bi-axially symmetric Helmholtz equation, Complex Variables and Elliptic Equations 52(8) (2007), 673-683.

[10] A. Hasanov and E. T. Karimov, Fundamental solutions for a class of threedimensional elliptic equations with singular coefficients, Appl. Math. Lett. 22 (2009), 1828-1832.

[11] A. Hasanov and H. M. Srivastava, Some decomposition formulas associated with the Lauricella function $F_{A}^{(r)}$ and other multiple hypergeometric functions, Appl. Math. Lett. 19(2) (2006), 113-121.

[12] A. Hasanov and H. M. Srivastava, Decomposition formulas associated with the Lauricella multivariable hypergeometric functions, Comput. Math. Appl. 53(7) (2007), 1119-1128.

[13] E. T. Karimov, On a boundary problem with Neumann's condition for 3D singular elliptic equations, Appl. Math. Lett. 23 (2010), 517-522.

[14] E. T. Karimov and J. J. Nieto, The Dirichlet problem for a 3D elliptic equation with two singular coefficients, Comput. Math. Appl. 62 (2011), 214-224.

[15] P. Kumar, Approximation of growth numbers generalized bi-axially symmetric potentials, Fasciculi Mathematics 55 (2005), 51-60. 
[16] G. Lauricella, Sulle funzione ipergeometriche a più variabili, Rend. Circ. Mat. Palermo 7 (1893), 111-158 (in Italian).

[17] P. A. McCoy, Polynomial approximation and growth of generalized axisymmetric potentials, Canadian Journal Mathematics 31(1) (1979), 49-59.

[18] J. J. Nieto and E. T. Karimov, On an analogue of the Holmgreen's problem for 3D singular elliptic equation, Asian-European Journal of Mathematics 5(2) (2012), 1250021, 18 pages.

[19] M. N. Olevskii, Solution of the Dirichlet problem for the equation for a hemispherical region, Dokl. Akad. Nauk SSSR (N.S) 64 (1949), 767-770 (in Russian).

[20] M. Rassias, Lecture Notes on Mixed Type Partial Differential Equations, World Scientific, 1990.

[21] M. S. Salakhitdinov and A. Hasanov, The Dirichlet problem for generalized biaxially symmetric Helmholtz equation, Eurasian Mathematical Journal 3(4) (2008), 99-110.

[22] M. S. Salakhitdinov and A. Hasanov, A solution of the Neumann-Dirichlet boundary value problem for generalized bi-axially symmetric Helmholtz equation, Complex Variables and Elliptic Equations 53(4) (2008), 355-364.

[23] M. S. Salakhitdinov and A. Hasanov, The boundary problem $N D_{1}$ for generalized axially symmetric Helmholtz equation, Reports of International Academy of Sciences of Adygey 13(1) (2012), 109-116.

[24] M. S. Salakhitdinov and E. T. Karimov, Spatial boundary problem with the DirichletNeumann condition for a singular elliptic equation, Appl. Math. Comput. 219 (2012), 3469-3476.

[25] R. J. Weinacht, Some properties of generalized axially symmetric Helmholtz potentials, SIAM J. Math. Anal. 5 (1974), 147-152.

Received May 13, 2019; revised August 26, 2019; accepted October 7, 2019.

\section{Author information}

Davlatjon R. Muydinjanov, Kokand State Pedagogical Institute named after Mukimi, Kokand, Fergana, Uzbekistan.

E-mail: davlatjon.kspi@mail.ru 